\title{
Die Kraft der Gedanken
}

Autosuggestionstechniken bei Stress - Die Persönlichkeit stärken, Eigenressourcen nutzen Claus Derra

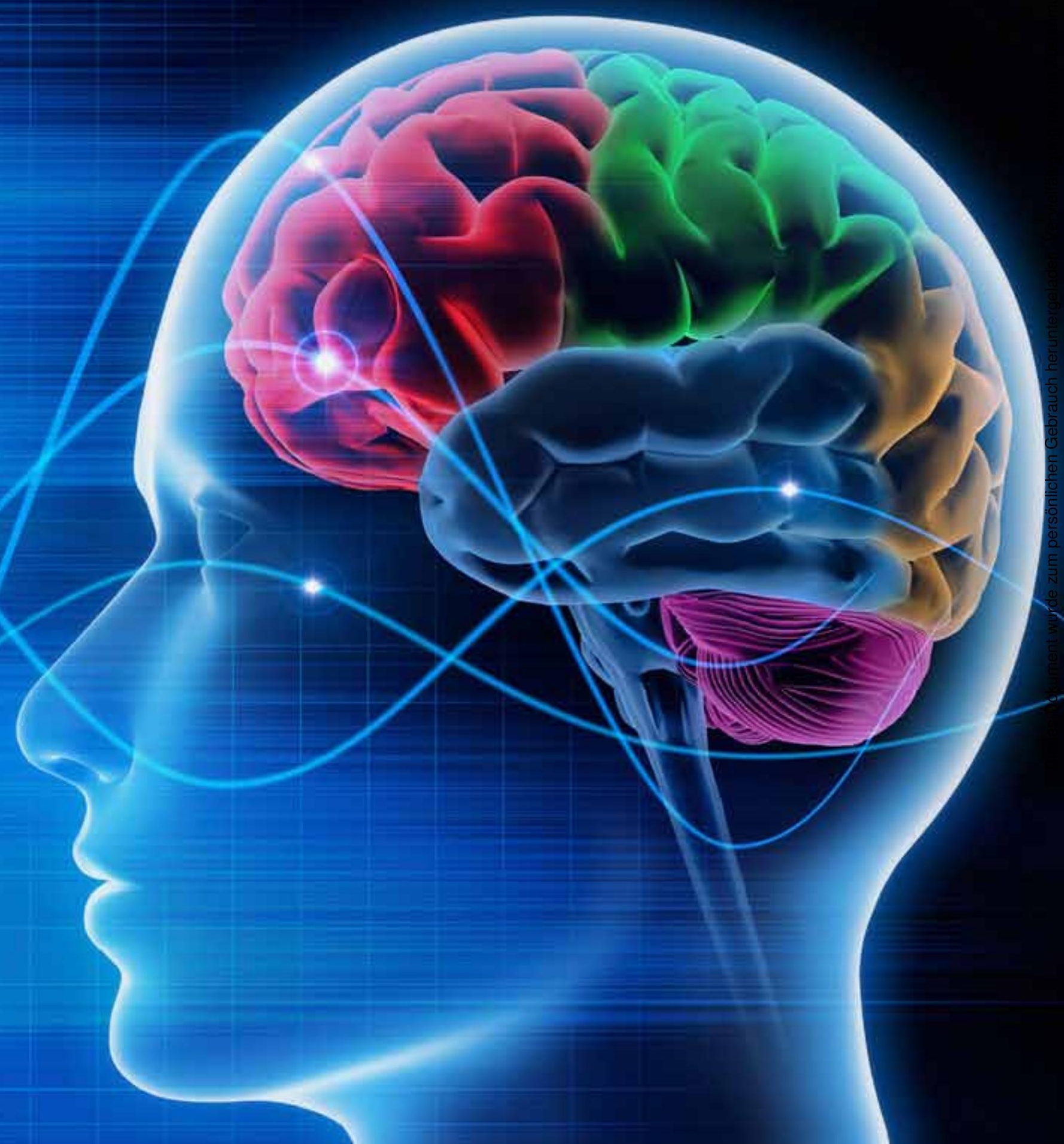




\section{Zusammenfassung}

Was für den einen eine angenehme Stimulation ist, kann für einen anderen schon Stress bedeuten, wie etwa laute Musik. Die Wahrnehmung eines Reizes als Stressor geht einher mit einer Bewertung und in der Folge, ob Bewältigungsstrategien zur Verfügung stehen, damit Stress nicht zum Gesundheitsrisiko wird.

Autosuggestionen können die Stressbewältigung wirkungsvoll unterstützen. Die autosuggestiv wirksamen Wörter und Sätze werden aus dem individuellen Erleben heraus gebildet, eingeübt, modifiziert, um dann in entsprechenden Stresssituationen eingesetzt werden zu können. Sie unterliegen einem Lernprozess, der durch ständige Wiederholungen zur Verstärkung, zur Aufnahme ins Unterbewusstsein und schließlich zur Automatisierung durch Konditionierung führt.

„Überleben im Stress“ heißt der Titel eines Buches von Hannes Lindemann, das 1973 in großer Auflage erschien [1]. Darin beschreibt der Arzt und Hochleistungssportler wie er 1956 mithilfe des Autogenen Trainings als erster in einem Serienfaltboot den Atlantik überquerte, ein Abenteuer, das vor ihm über 100 Menschen mit dem Leben bezahlten. Bei einer früheren Atlantiküberquerung in einem Einbaum hatte er die Erfahrung gemacht, dass er physisch, technisch und navigatorisch gut vorbereitet war, psychisch dagegen nicht. Daher hatte er sich nun monatelang mit dem Autogenen Training auf diese Extremleistung vorbereitet. „Wer sich autogen entspannt, spart Kraft und Kalorien; er lebt ökonomischer als der verkrampfte Mensch. Tiefstes Entspannen führt zu Wohlbehagen. Wer sich richtig entspannen kann, vergisst seine naturgegebene Angst. Das Schlafbedürfnis nimmt ab, die Sitzunruhe lässt nach, man sitzt so entspannt, dass es nicht so schnell zu Sitzbeschwerden kommt.“ [1].

Als entscheidend für den erfolgreichen Abschluss sah Lindemann jedoch die Arbeit mit Autosuggestionen. Durch das monatelange Einüben der formelhaften Vorstellungen „Kurs West“ und „Ich schaffe es“ war er mental in der Lage, die in der Einsamkeit zwangsläufig auftretenden Halluzinationen zu durchbrechen und immer wieder seine wache Reaktionsfähigkeit zum Überleben herzustellen.

\section{Die Kraft der Gedanken}

Zugegeben ist das genannte Beispiel sicherlich nicht für unseren Alltag geeignet, sondern eher eine außergewöhnliche Form von differenzierter Stressbewältigung extremer physischer und psychischer Herausforderungen. Lindemann konnte damit jedoch die „Kraft der Gedanken“, die Möglichkeiten der Einflussnahme auf eigene Gefühle, Gedanken und Verhalten durch die systematische Entwicklung der persönlichen Vorstellungskraft belegen. Seither ist das Autogene Training lange Zeit synonym für erfolgreiche Stressbewältigung gewesen. Heute haben wir durch die Verhaltenstherapie, das mentale Training und das sog. positive Denken weitere systematische Ansätze der Stressbewältigung durch Anwendung von Autosuggestionen entwickelt.

Die Wirksamkeit von Suggestionen zur Beeinflussung von psychischer Befindlichkeit ist seit Jahrhunderten bekannt. Ärzte und Heiler haben schon im Altertum Krankheiten mit suggestiven Ele- menten behandelt. Schamanen wussten immer schon, dass Sätze und bildhafte Vorstellungen besonders wirksam werden, wenn sie vorher einen Trancezustand induzieren.

Als Begründer der modernen bewussten Autosuggestion wird der französische Apotheker Emile Couè (Nancy 1857-1926) angesehen. Couè stellte fest, dass es einen großen Unterschied macht, mit welchen Worten er seinen Patienten die vom Arzt verschriebenen Medikamente überreichte. Gab er seinen Patienten ein Medikament mit den Worten „Da hat Ihnen Ihr Arzt ein sehr gutes Mittel verschrieben. Sie werden sehen, dass es Ihnen bald besser geht“ dann ging es vielen nach kurzer Zeit besser oder sie waren geheilt. Gab er den Patienten das Medikament jedoch ohne solche die Wirksamkeit des Medikaments lobenden Worte, dann berichteten die Patienten viel seltener über eine Besserung ihrer Beschwerden [2]. Entscheidend dabei war jedoch nicht der gesprochene Satz, sondern die Frage, wie die Botschaft beim jeweiligen Patienten ankam, ob er den Inhalt also autosuggestiv umsetzen konnte.

Auch die moderne Hypnotherapie [3] und das Autogene Training [4] fokussieren heute weniger die Person des Therapeuten, sondern die Frage, was von den Interventionen des Therapeuten vom Pati-

\section{Eine Person erleidet eine Stressreaktion}

\section{nicht einfach passiv, sondern reagiert als Individuum auf den Stressor. Die Stress-} bewältigung wird durch Wahrnehmung und Bewertung begleitet.

enten aufgenommen und autosuggestiv verarbeitet wird - d. h. jede Suggestion wirkt letztendlich über Autosuggestion.

Heutzutage sind mentale Techniken zur Leistungssteigerung im Sport, im Beruf, aber auch im kreativen Bereich zur Selbstverständlichkeit geworden und durch viele Fallbeispiele sowie wissenschaftliche Studien gut belegt [5].

\section{Wirkerwartungseffekte}

Interessanterweise hat uns in der Erforschung der Grundlagen der Autosuggestion ein Bereich in den letzten Jahren neue Erkenntnisse gebracht, der von der Medizin eher skeptisch und geringschätzend betrachtet wird - die Untersuchung von sog. Wirkerwartungseffekten in der Placeboforschung.

Wirkerwartungseffekte sind Autosuggestionen, die nur wirksam werden können, wenn der Betroffene über eine Intervention informiert ist. Danach entstehen unterschiedliche Wirkerwartungseffekte schon allein durch Faktoren wie z.B. die äußere Form eines Medikaments oder die Art einer Intervention: Kleine oder besonders große Pillen wirken besser als normal große, mehrfach teilbare Tabletten haben eine höhere Wirksamkeit, da sie eine hohe Wirkstoffdichte suggerieren, die bspw. auch noch in einer Vierteltablette wirkt.

Es gibt auch eine Rangreihe von Wirkerwartungseffekten abhängig von der Art der Intervention: Operationen haben die höchsten Effekte, dann kommen Spritzen, Pflaster, Tropfen und am Ende 


\section{Transaktionales Stressmodell}

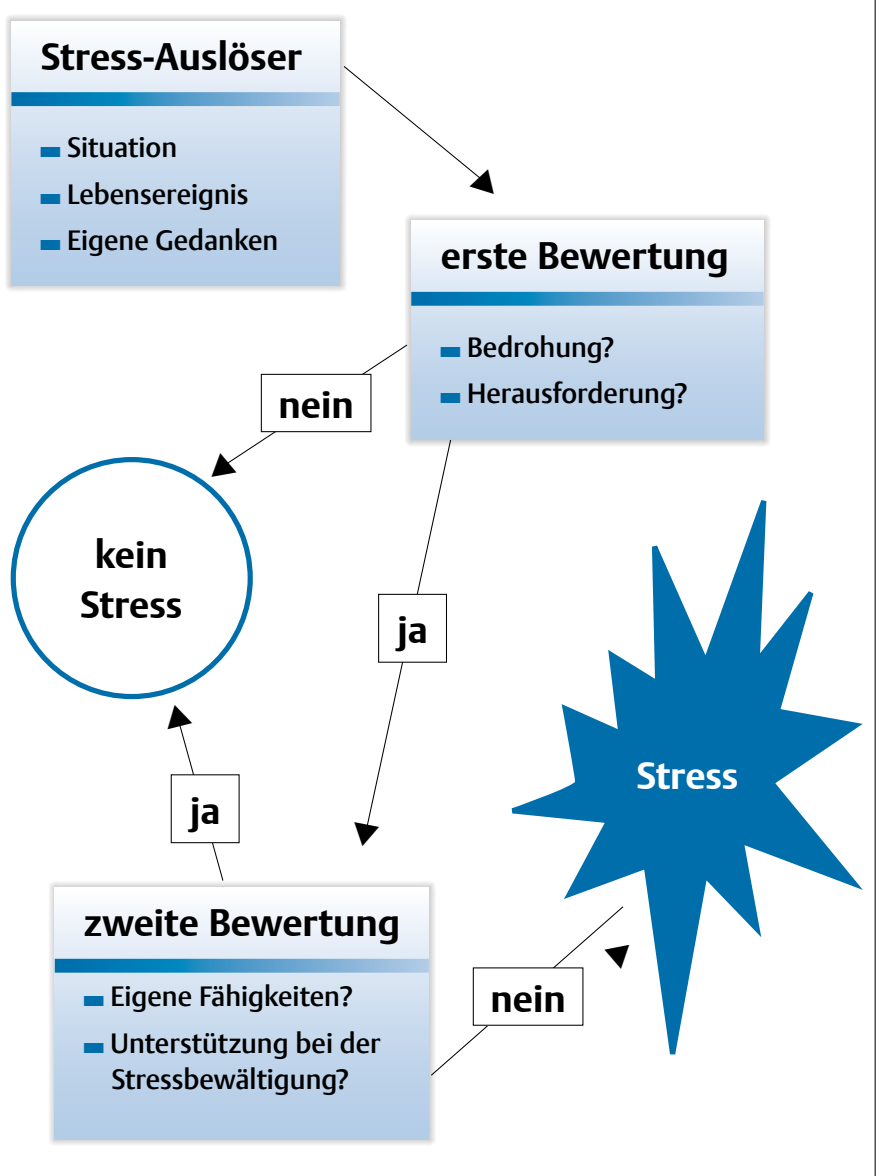

Abb. 1 Stressbewältigung. (in Anlehnung an [13])

\section{Stressbewältigung hängt ab von der Art des}

\section{Stressors, der Belastungsdauer und -stärke, der Kontrollierbarkeit der Situation und}

\section{den verfügbaren Bewältigungsstrategien.}

Tabletten. Je invasiver eine Maßnahme, desto höher ist die Erwartung an den Erfolg [6].

Die entscheidenden Variablen der Wirkerwartung sind jedoch die Art und Weise wie der Arzt etwas vermittelt und in welcher Form der Betroffene dies aufnimmt. Schon vor 15 Jahren wurden von der Turiner Arbeitsgruppe um Benedetti umfängliche Studien mit modifizierten verbalen Instruktionen durchgeführt [7]. Je nach Instruktion konnte die Wirkerwartung von Placebointerventionen in der Intensität und auch hinsichtlich der Wirksamkeit in bestimmten Körperbereichen gesteuert werden. Auch Noceboeffekte (Vorhersage von Nebenwirkungen) und sogar die Suggestion von Wirkungslosigkeit von Verumpräparaten wurden erzielt. In weiteren Studien konnte differenziert werden, dass Instruktionen einerseits über Suggestionen wirken [8], insbesondere aber auch über Stressreduktion [9]. Gerade der Einfluss auf die Stressreduktion scheint eine besonders wichtige Komponente der Wirkerwartung. Bei der Stressreduktion rückt gleichzeitig die Person des Arztes und Therapeuten wieder mehr in den Mittelpunkt. Besonders günstig wirken optimistische Persönlichkeiten mit hohen empathischen Qualitäten. Die Wirksamkeit der Autosuggestion wird also beeinflusst durch:

- die vermittelnde Person,

- die Art der Botschaft (Worte, Bilder),

- den Inhalt und die Richtung der Botschaft,

- die Aufnahmebereitschaft beim Empfänger sowie

- die autosuggestive Verarbeitung und emotionale Wirkung

beim Empfänger z.B. in Form von Stressreduktion.

\section{Wiederholung der Autosuggestion}

Eine weitere wichtige Variable der Wirksamkeit ist die Wiederholung der Autosuggestion. Eine Botschaft kann zwar schon nach einmaliger Vermittlung aufgenommen werden und weiterwirken. Wie wir aus der Psychotherapieforschung wissen, benötigen wir jedoch üblicherweise einige Erinnerungen oder immer erneute Wiederholungen bis die Inhalte autosuggestiv wirksam werden. Autosuggestionen unterliegen somit einem Lernprozess, der durch ständige Wiederholungen zur Verstärkung, zur Aufnahme ins Unterbewusstsein und schließlich zur Automatisierung durch Konditionierung führt.

Bei der Konditionierung wird ein Wort oder ein Bild zu einem Signal, das automatisch eine bestimmte Reaktion auslöst. So kann z. B. durch einiges Üben der Blick auf ein bestimmtes Bild an der Wand des Arbeitszimmers, eine rhythmische Bewegung oder auch nur ein tiefer Atemzug zur reflexartigen Entspannung und Stressreduktion eingesetzt werden, ohne dass wir uns dessen dauernd bewusst werden müssen. Auch der eingangs erwähnte Lindemann hat seine Autosuggestionen monatelang eingeübt, damit sie tief im Unterbewusstsein wirken konnten.

\section{Autosuggestion zur Bewältigung von chronischem Stress}

Schon Epiktet formulierte ca. 100 n. Chr.: „Es sind nicht die Dinge selbst, die uns beunruhigen, sondern die Vorstellungen und Meinungen von den Dingen.“ [10]. Die Mehrzahl von Stresssituationen, denen wir heute ausgesetzt sind, beziehen sich nicht auf einfache Stressoren, sondern auf psychosoziale Faktoren (Konflikte, Lärm, Befürchtungen, Kränkungen, Ärger, Überforderung, Erschöpfung u.ä.), oft sind andere Menschen mit einbezogen.

\section{Transaktionales Stressmodell}

Als wichtige und plausible Grundlage für Stressbewältigung durch Autosuggestion in solchen komplexen Situationen haben sich die Forschungsergebnisse der kognitiven Stresstheorien erwiesen, die v.a. mit dem Namen der Verhaltenstherapeuten Ross S. Lazarus [11] und Donald Meichenbaum [12] verbunden sind. Der grundlegende Gedanke der kognitiven Stresstheorien beinhaltet, dass eine Person eine Stressreaktion nicht einfach passiv erleidet, sondern als Individuum auf den Stressor reagiert und es dadurch zu einem Interaktionsprozess kommt, der durch Wahrnehmung und Bewertung die eigentliche Stressbewältigung begleitet (transaktionales Stressmodell [11]).

So zeigte man Versuchspersonen sehr realistische Filme mit detaillierten Szenen und Nahaufnahmen von Unfällen und gab vorher 
entweder gar keine oder ganz unterschiedliche Informationen darüber. Man konnte feststellen, dass die Stressreaktionen der Zuschauer sehr davon abhingen, welche Erwartungen entstanden waren. Es zeigte sich, dass sich nicht jede Vorinformation günstig auf die Stressreaktion auswirkte. Auch in Studien mit Patienten vor Operationen wurde untersucht, wie die Information vor der Operation den späteren Heilungsverlauf beeinflusste. Stress ist daher nach Lazarus die Antizipation (d.h. gedanklich in der Vorstellung vorweggenommen) einer Gefährdung oder Bedrohung der eigenen Person angesichts einer ersten Bewertung wahrgenommener Situationen.

Ist die Bedrohlichkeit gegeben, entsteht im Rahmen der sog. sekundären Bewertung eine Auswahl möglicher Bewältigungsstrategien. Darauf erfolgt in einer Art Feedbackschleife eine Neubewertung oder Wiederbewertung der Situation im Hinblick auf die Konsequenzen des eigenen Handelns. Abb. 1 zeigt eine vereinfachte Darstellung dieser Prozesse, die schnell plausibel macht, wie Veränderungen geplant werden können.

Auf einen Reiz reagieren wir immer mit einer ersten Bewertung, die zunächst darüber entscheidet, ob wir ihn überhaupt als Stressor wahrnehmen. Beispielsweise wird ein Jugendlicher in der U-Bahn durch die Heavy Metal-Musik seiner Lieblingsband im Kopfhörer seines MP3-Players angenehm stimuliert, während der ältere Herr nebendran schon durch das sehr abgedämpfte Hören der Rhythmen völlig genervt ist. Die Frage, ob der Stressor eine Bedrohung oder Herausforderung darstellt, leitet automatisch zur zweiten Bewertung über: „Habe ich Strategien, um die Situation zu beeinflussen? Kann ich mir Hilfe holen?“ Stehen mir solche Stressbewältigungsstrategien zur Verfügung, wird sich der Stress kaum intensiv auswirken können. Ist das jedoch nicht der Fall, sind wir mit der Bewäl- tigung überfordert (und ein solches Gefühl entsteht in uns Menschen recht schnell), dann entsteht Stress, der sich gesundheitlich ungünstig auswirken kann.

Transaktionale Stressmodelle haben die früheren physiologischen Modelle wesentlich dadurch ergänzt, dass sie deutlich machen, dass eine neue Sichtweise das körperliche Erregungsniveau nachhaltig positiv oder negativ beeinflussen kann. Denk- und Gefühlsprozesse rückten damit mehr ins Zentrum der Stressbewältigungsstrategien.

\section{Persönliche Strategien der Stressbewältigung}

Stressbewältigung hängt also von der Art des Stressors, von Dauer und Stärke der Belastung, der Kontrollierbarkeit der Situation und den zur Verfügung stehenden Bewältigungsstrategien ab. Eine Person wird eine Stresssituation dann gut bewältigen, wenn sie in der Bewertung zum Ergebnis kommt, dass ihre Fähigkeiten dazu ausreichen. Die meisten Menschen haben Erfahrung mit Stress und i.d.R. auch mehr oder weniger wirksame persönliche Strategien entwickelt. Für den akuten Stress haben sich insbesondere bewährt:

- Kurzübungen zur Entspannung,

- Wahrnehmungsablenkung weg vom Stress auf angenehme

Erlebnisse,

- positive Selbstgespräche („du schaffst das schon“) oder

- körperliche Abreaktion.

Alle diese Strategien werden in ihrer Effektivität verstärkt, wenn sie durch autosuggestive Bewertungen begleitet werden. Ein Mitarbeiter eines Ministeriums hatte sich bspw. überlegt, die Abreaktion nicht in seinem Büro durchzuführen, sondern sich eine Akte zu nehmen, den Raum zu verlassen und dann mit wichtigem
Abb. 1 Positive Kraftquellen werden im gedanklichen Kontakt mit dem „inneren Hefter“ bewusst.

Gesicht in schnellem Schritt durch die Gänge des Ministeriums zu laufen. Keiner wagte ihn anzuhalten. Autosuggestiv verstärkte er die Wirksamkeit, indem er sich freute, dass alle, die ihm begegneten, denken mussten, er wäre auf dem Weg zu einer wichtigen Besprechung. So kann aus der allgemeinen Empfehlung „Abreaktion“ eine ganz persönliche Strategie mit autosuggestiver Verstärkung werden.

\section{Strategien in komplexen Stresssituationen}

Wenn es um komplexere und länger andauernde Stresssituationen geht, werden solche einfachen Strategien nicht ausreichen. Am sinnvollsten wäre es, in solchen Situationen mit einem Fachmann (Psychotherapeut, Psychologe, geschulter Stressberater o.ä.) zu sprechen, um eine genaue Analyse der Einflussfaktoren und Änderungsmöglichkeiten zu ermöglichen.

Wenn dies nicht möglich ist, empfiehlt sich das Gespräch mit einem sog. inneren Beobachter oder inneren Helfer. Man nimmt sich etwas 


\section{Sieben Regeln zur Bildung autosuggestiv wirksamer Worte und Sätze:}

1. Sie sollten kurz, prägnant und klar sein (wie in der Werbung).

2. Sie sollten das Problem und seine Veränderung möglichst präzise erfassen.

3. Sie sollten positiv formuliert sein (möglichst keine Verneinungen).

4. Sie sollten persönlichkeitsnah sein, d. h. der persönlichen Umgangssprache (ggf. Dialekt oder Muttersprache) entsprechen.

5. Sie sollten wahr sein (keine unrealistischen Ziele).

6. Sie sollten in möglichst einfacher und konkreter Sprache formuliert sein (keine nichtssagenden, abstrakten Begriffe).

7. Nicht zu schnell Wirksamkeit erwarten, Geduld bei der Anwendung.

Zeit, setzt sich an einen ruhigen Ort und nimmt in der Vorstellung Kontakt mit dem inneren Beobachter auf. Dieser kann eine Person sein, die man gut kennt und zu der eine gute Beziehung besteht. Es kann aber auch ein Fantasiewesen sein. Wichtig ist das innere Gefühl, mit einem Gegenüber reden zu können und Fragen zu erörtern wie z.B.:

- was genau ist das Problem?

- welche Personen spielen eine Rolle?

- was stört oder belastet mich?

- was möchte ich verändern?

- was soll stattdessen sein?

- welches Ziel strebe ich an?

Es empfiehlt sich, die Gedanken nach einer solchen kurzen Meditation aufzuschreiben und sich ähnlich wie bei einem Brainstorming Notizen zu machen. Aus diesen Notizen können dann erste Ideen für Worte oder Sätze zur autosuggestiven Stärkung der Persönlichkeit entwickelt werden.

Eines muss dabei immer klar sein: Autosuggestiv können wir nur auf uns selbst Einfluss nehmen, die Umgebung ändern wir dadurch natürlich nicht. Wir können aber unsere Persönlichkeit so stärken, dass wir dadurch den Mut finden, aktiv unsere Umgebung zu ändern. Manchmal ist es schwierig, alleine auf gute Ideen zur Stärkung der Persönlichkeit zu kommen. Hier könnte man durchaus auf Ratgeber zur Stressbewältigung, auf entsprechende Stichworte im Internet oder auch auf beratende Hörbücher zurückgreifen. Die gesprochene Stimme kann den Dialog erleichtern. Eine gute Anleitung zum Bilden von Leitsätzen im Rahmen des Autogenen Trainings findet sich unter [14].

\section{Vorgehen in der Anwendung autosuggestiver Techniken}

Die Vorgehensweise bei der Anwendung von autosuggestiven Techniken besteht in mehreren Schritten: Entwickeln, Wiederholen, Modifizieren, Einsetzen.

\section{Entwickeln}

Zunächst wird ein erster Entwurf der Autosuggestion z. B. aus den Notizen hergestellt. Worte können dabei von bildhaften Vorstellungen begleitet sein und damit verbunden werden, sodass die Wirksamkeit verstärkt wird [15].
Aus diesem Rohentwurf erfolgt dann die Entwicklung von geeigneten und persönlich wirksamen Worten und Sätzen durch inneres Wiederholen in entspannten Situationen. Zu Beginn stehen z. B. die Begriffe „Erschöpfung“ und „Schlafmangel“. Im weiteren Verlauf der Überlegungen werden positive Begriffe wie z. B. „Kraft“ und „Ruhe“ gegenübergestellt und daraus Autosuggestionen formuliert wie „Ich spüre meine Kraft und finde meine Ruhe“.

\section{Wiederholen}

Dieser Satz wird nun als eine Art Tagesbegleiter immer wieder im entspannten Zustand innerlich formuliert und wiederholt.

\section{Modifizieren}

Eine weitere persönliche Modifikation könnte dann in den Satz münden „Die Kugel der Ruhe findet die Mitte der Schale“.

\section{Einsetzen}

Dieser Satz, der einen sehr persönlichen Bezug zu eigenen Erlebnissen haben kann, wird dann immer wieder memoriert und geübt, bis er dann in entsprechenden Stresssituationen wirksam eingesetzt werden kann.

Autosuggestive Techniken können bei Stress sehr wirksam sein. Wir alle kennen die stressverstärkende Wirkung von negativen Autosuggestionen, die bei Überforderung automatisch in uns entstehen („Ob das wohl gut geht ... das schaffe ich nicht ... wenn das so weitergeht ... alle wollen was von mir und keiner denkt an mich ...“). Die gezielte positive Entwicklung ist daher nicht leicht, oft empfiehlt sich das Gespräch mit einem Therapeuten zur professionellen Beratung. Der entscheidende Punkt ist jedoch die Geduld bei der Anwendung und das Vertrauen in die Kraft der eigenen Gedanken. Wer diese Techniken für sich alleine entwickeln will, muss nicht Sorge haben, dass er möglicherweise etwas falsch macht. Es kann eigentlich nichts passieren außer, dass die Sätze nichts von der erwarteten Wirkung zeigen, wenn man z.B. die o.g. Regeln nicht berücksichtigt.

Autosuggestion ist eine gute Möglichkeit zur Stärkung der eigenen Ressourcen, sollte aber klug eingesetzt werden, wie das Sprichwort sagt:

Ändere die Dinge, die Du ändern kannst.

Akzeptiere Dinge, die Du nicht ändern kannst.

Und lerne zwischen beiden zu unterscheiden.

Derjenige, dem dieses gelingt, wird feststellen, wie erstaunlich leicht sich mancher Stress durch etwas mehr Gelassenheit auch ohne Autosuggestion auflösen lässt.

Interessenkonflikt: Der Autor erklärt, dass keine wirtschaftlichen oder persönlichen Verbindungen bestehen.

Online zu finden unter

http://dx.doi.org/10.1055/s-0035-1545366

\section{Literatur}

1 Lindemann H. Überleben im Stress - Autogenes Training. München: Bertelsmann; 1973

2 Coue E. Die Selbstbemeisterung durch bewußte Autosuggestion. Basel: Benno Schwabe; 1922 
$\overline{3}$ Revenstorf D. Hypnotherapie. Expertise zur Beurteilung der wissenschaftlichen Evidenz des Psychotherapieverfahrens. Tübingen; 2003. Im Internet: http://www.dgh-hypnose.de/wissenschaftliche-anerkennung.html

$\overline{4}$ Thomas K. Praxis des Autogenen Trainings. Stuttgart: Trias; 2006

5 Hoffmann B. Handbuch Autogenes Training. München: DTV; 2012

$\overline{6}$ Jäger B, Lamprecht $\mathrm{F}$. Zum Verständnis von Placebowirkungen bei Schmerzpatienten. In: Egle UT, Lehmann KA, Hoffmann SO et al. Hrsg. Handbuch chronischer Schmerz. Stuttgart: Schattauer; 2003

7 Amanzio M, Benedetti F. Neuropharmacological dissection of placebo analgesia: expectation-activated opioid systems versus conditioningactivated specific subsystems. J Neurosci 1999; 19 (1): 484-494

8 De Pascalis V, Chiaradia C, Carotenuto E. The contribution of suggestibility and expectation to placebo analgesia phenomenon in an experimental setting. Pain 2002; 96 (3): 393-402

9 Pollo A, Vighetti S, Rainero I et al. Placebo analgesia and the heart. Pain 2003; 102 (1-2): 125-133

$\overline{10}$ Epiktet (um 50 n. Chr. - 138), griechisch: Epiktetos, griechischer Stoiker und Philosoph.

$\overline{11}$ Lazarus RS, Folkman S. Stress, appraisal and coping. New York: Springer; 1984

$\overline{12}$ Meichenbaum D. Intervention bei Stress - Anwendung und Wirkung des Stressimpfungstrainings. Bern: Huber; 1991

$\overline{13}$ VDR. Aktiv Gesundheit fördern - Gesundheitsbildungsprogramm der Rentenversicherung für die medizinische Rehabilitation. Stuttgart: Schattauer; 2000 $\overline{14}$ Derra C. Ziele erreichen. Autogenes Training für Könner. Hörbuch. Stuttgart: Trias; 2010

$\overline{15}$ Hüther G. Die Macht der inneren Bilder. Göttingen: Vandenhoeck und Ruprecht; 2005

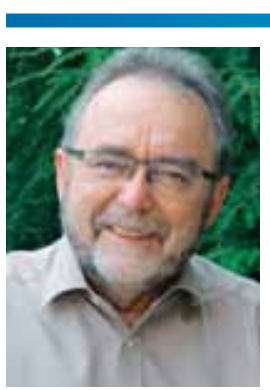

Dr. med. Dipl.- Psych. Claus Derra

Josef-Weiß-Str. 6

97980 Bad Mergentheim

derra@gmx.de

Claus Derra ist Facharzt für Psychiatrie und Psychosomatik.

Er war ärztlicher Direktor eines Rehabilitationszentrums der Deutschen Rentenversicherung in Bad Mergentheim. Seine Schwerpunkte in Forschung und Behandlung sind Schmerztherapie, Entspannungsverfahren und Hypnose. Er hat mehrere Bücher und Hörbücher zu Entspannungsverfahren und Stressbewältigung durch Achtsamkeit veröffentlicht. 